\title{
Acute Liver Failure in a Patient Travelling From Asia: The Other Face of the Coin of Infectious Disease
}

\author{
Balen Abdulrahman ${ }^{\mathrm{a}}$, Mohamed H. Ahmed ${ }^{\mathrm{b}, \text { e }}$, John Ramage ${ }^{\mathrm{c}, \mathrm{d}}$
}

\begin{abstract}
We present a case of a 63-year-old male who had travelled from South India to United Kingdom (UK) visiting relatives. He had developed episodes of diarrhea, vomiting and fevers while travelling and on assessment in hospital, mild abdominal distension was noted with rapid deterioration to hypovolemic shock. Initial blood test showed a low platelet count with deranged liver function tests (LFTs). It was noted that during admission to intensive care unit (ICU), blood continued to ooze from a previous surgical laparoscopy wound, central and arterial line access sites. Blood results revealed ongoing derangement of clotting and LFT. Computed tomography (CT) scan showed possible acute cholecystitis and a laparoscopy showed an ischemic-looking liver and gut but no significant gallbladder abnormality. The virology screen was positive for dengue virus antibodies IgM and IgG. The patient developed multi-organ failure and deteriorated despite intensive support. Post mortem showed fulminant hepatic failure and acute tubular necrosis of kidneys.
\end{abstract}

Keywords: Liver failure; Hepatitis; Dengue disease; Coagulation

\section{Introduction}

First onset of acute liver failure in patients with history of recent travel to tropical countries will suggest the need to exclude infectious diseases as an initial hypothesis. Importantly, delay in diagnosis may lead to an increase in mortality and morbidity. Dengue disease can be associated with acute liver failure.

Manuscript submitted May 11, 2017, accepted June 12, 2017

aDepartment of Cardiology, Milton Keynes University Hospital NHS Foundation Trust, Eaglestone, Milton Keynes, Buckinghamshire, UK

bepartment of Medicine and HIV Metabolic Clinic, Milton Keynes University Hospital NHS Foundation Trust, Eaglestone, Milton Keynes, Buckinghamshire, UK

'Liver Unit in Kings College Hospital, London, UK

dBasingstoke and North Hampshire Hospital, Basingstoke, UK

${ }^{\mathrm{e}}$ Corresponding Author: Mohamed H. Ahmed, Department of Medicine and HIV Metabolic Clinic, Milton Keynes University Hospital NHS Foundation Trust, Eaglestone, Milton Keynes, Buckinghamshire, UK.

Email: elziber@yahoo.com

doi: https://doi.org/10.14740/gr856w
Dengue fever (DF) is mosquito born disease caused by any one of four related single-stranded RNA flavivirus from flaviviridae family $[1,2]$. This virus is transmitted to humans by the Aedes aegypti mosquito. The incubation period is 3 - 14 days. Dengue has a disease burden estimated to be 1,600 disabilityadjusted life years per million population, which is similar to tuberculosis [3]. As a tropical disease, dengue is deemed only second in importance to malaria [4], though the World Health Organization counts dengue as one of 16 neglected tropical diseases [5]. The disease is endemic in more than 100 tropical and subtropical countries, comparable to malaria area distribution [2]. Therefore, in the era of easy travel across continents, we believe it is crucial that acute and emergency medicine physicians should familiarize themselves with all common tropical disease manifestations.

\section{Case Report}

A 63-year-old male was travelling with his wife from India to UK. The patient became unwell, malaise and diarrhea whilst on the flight. Upon arrival to the UK, he continued feeling lethargic, with a loss of appetite, experiencing fevers and rigors. The patient reported loose stools up to three times a day, with no blood. Past medical history showed mild asthma and type 2 diabetes mellitus controlled by diet.

Two days after his arrival in the UK, he was admitted to the emergency department. Initial examination showed mild abdominal distension and tenderness in the right upper quadrant of the abdomen. Blood pressure was $85 / 55 \mathrm{~mm} \mathrm{Hg}$, pulse was $87 \mathrm{bpm}$, oxygen saturation was $98 \%$ on room air and temperature was $36^{\circ} \mathrm{C}$. His platelet count was low $\left(7 \times 10^{9} / \mathrm{L}\right)$, with normal white cell counts (WCC) and hemoglobin with mild renal failure. Liver function tests (LFTs) were slightly deranged (Table 1). A marked metabolic acidosis on arterial blood gas analyses was noted. Hepatitis screen was sent soon after admission and result was available next day: negative for $\mathrm{A}, \mathrm{B}$ and $\mathrm{C}$ viruses, negative for malaria parasite, and negative for influenza $\mathrm{A}(\mathrm{H} 1 \mathrm{~N} 1)$ virus. Chest and abdominal radiography were normal. The rest of virology screen was sent on day 3 and result was available after the death of the patient. The virology screen was positive for dengue virus antibodies IgM and IgG (Table 2). Subsequent overnight review revealed bleeding from mouth and urgent platelet transfusion was arranged. The patient remained hypotensive. An urgent $\mathrm{CT}$ of abdomen was requested (Figs. 1 and 2). This was followed by 
Table 1. Summary of Biochemistry and Complete Blood Count Results

\begin{tabular}{llll}
\hline Blood & Day 1 & Day 2 & Day 3 \\
\hline $\mathrm{Hb}(\mathrm{g} / \mathrm{L})$ & 89 & 96 & 76 \\
WCC $\left(10^{9} / \mathrm{L}\right)$ & 3 & 4.2 & 4 \\
Platelet $\left(10^{9} / \mathrm{L}\right)$ & 7 & 15 & 17 \\
Neutrophils $\left(10^{9} / \mathrm{L}\right)$ & 2.1 & 2.9 & 2.3 \\
Lymphocytes $\left(10^{9} / \mathrm{L}\right)$ & 0.3 & 0.6 & 0.5 \\
Albumin $(\mathrm{g} / \mathrm{L})$ & 28 & 31 & 29 \\
Alkaline phosphatase $(\mathrm{IU} / \mathrm{L})$ & 197 & 180 & 169 \\
ALT $(\mathrm{IU} / \mathrm{L})$ & 2,363 & 2,136 & 2,691 \\
Bilirubin $(\mu \mathrm{mol} / \mathrm{L})$ & 26 & 31 & 46 \\
Urea $(\mathrm{mmol} / \mathrm{L})$ & 20.9 & 21.1 & 18.8 \\
Creatinine $(\mu \mathrm{mol} / \mathrm{L})$ & 236 & 248 & 262 \\
Sodium $(\mathrm{mmol} / \mathrm{L})$ & 134 & 134 & 141 \\
\hline Potassium $(\mathrm{mmol} / \mathrm{L})$ & 4.5 & 4.8 & 4.8 \\
Glucose $(\mathrm{mmol} / \mathrm{L})$ & 22.1 & 17.1 & 6.2 \\
Prothrombin time $(\mathrm{s})$ & 17.9 & 22.8 & 28.6 \\
\hline
\end{tabular}

an urgent diagnostic laparoscopy to exclude a gangrenous gallbladder. At laparoscopy, a large amount of fluid in peritoneum was seen, with an inflamed and swollen liver.

After laparoscopy, the patient was transferred to intensive care unit (ICU), required circulatory and respiratory support with initiation of hemofiltration. Despite transfusion of two units of platelet and four units of fresh frozen plasma, there was continuous oozing of blood from mucosal surfaces in association with anuric renal failure. Importantly, marked abdominal distension was noted, with a minimum response to maximal organ support. The patient deteriorated and died $72 \mathrm{~h}$ after admission. Post mortem showed fulminant hepatic failure and acute tubular necrosis of kidneys.
Table 2. Summary of Virology Screen Results

\begin{tabular}{ll}
\hline Blood & Status \\
\hline Hantavirus & Negative \\
Saaremaa virus IgG & Not detected \\
Leptosira & Negative \\
Chikungunya & Negative \\
Sindbis & Negative \\
Alphavirus & Negative \\
Rickettsia serology & Negative \\
Epidemic typhus group & Negative \\
Tsutsugamushi & Negative \\
Sand fly fever & Negative \\
Cyprus virus & Negative \\
West Nile & Negative \\
Dengue virus IgM & Positive \\
Dengue virus IgG & Positive \\
\hline
\end{tabular}

\section{Discussion}

There is a huge increasing incidence of DF globally. It is estimated to be 50 - 100 million cases per year with most cases occurring in Asia and South America [2]. Dengue causes four spectra of illness: asymptomatic phase, acute febrile illness, classic DF and dengue hemorrhagic fever (DHF) which includes dengue shock syndrome (DSS) $[6,7]$. Liver involvement in DF includes hepatomegaly and deranged liver enzyme [8]. Transient variable liver function abnormalities are found in most of patients with symptomatic DF. This can be due to direct viral infection of hepatocytes or consequences of body immune response against virus causing deranged LFTs.

The association of hepatic failure to DHF/DSS was reported in epidemics of DF in 1970 in Indonesia, later on in the

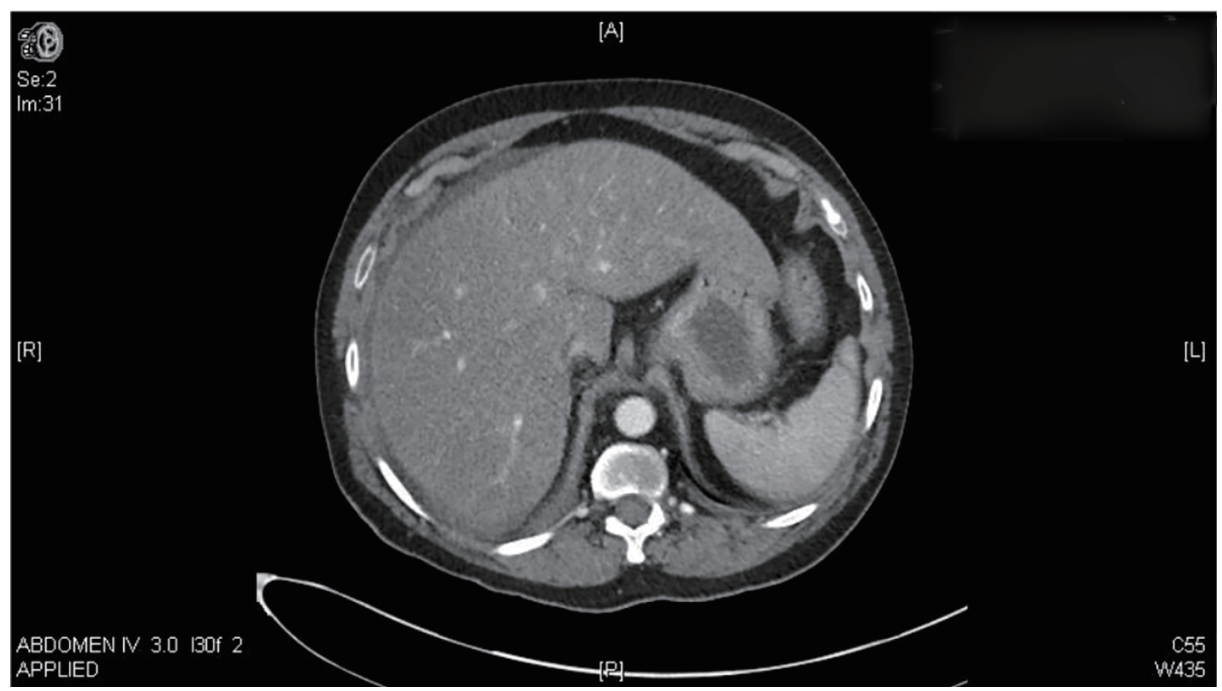

Figure 1. Transverse view of CT of abdomen. 


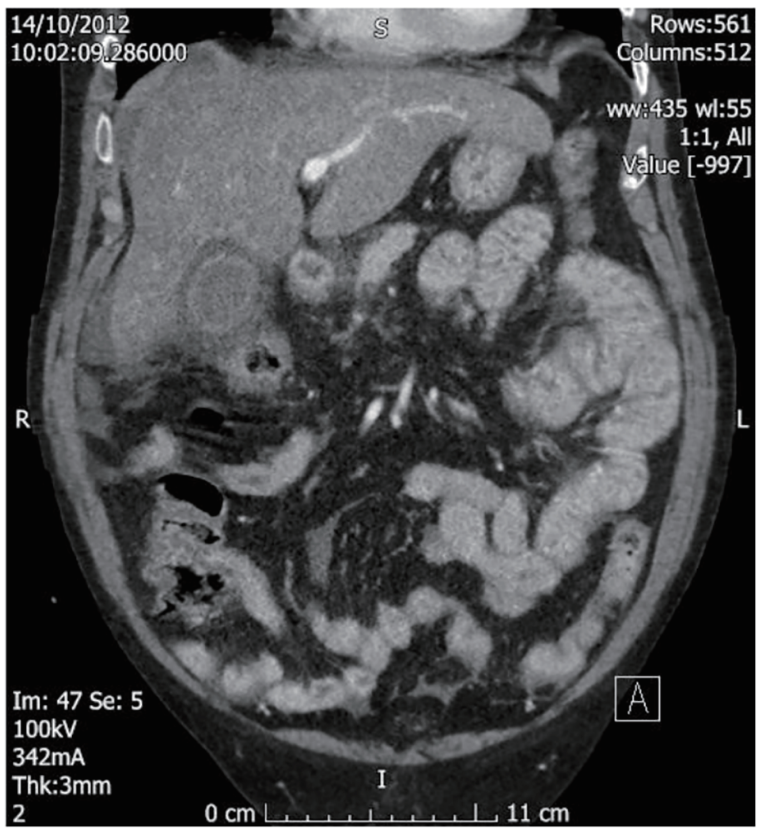

Figure 2. Longitudinal view of CT of abdomen. CT showed inflammatory changes around gall bladder likely gall bladder wall thickening or right colon ischemia.

1987 epidemic in Thailand and 1990 in Malaysia [9]. Several studies indicate poor prognosis of fulminant hepatic failure from DF [10-12]. The pattern of liver enzyme elevation appears to be mild to moderate in most cases of DF and AST raises higher than ALT levels [13].

The case presented had primary DF infection with acute hepatic failure and severe thrombocytopenia. Other diseases with similar presentation were ruled out including complicated malaria, leptospirosis and viral hepatitis. Pointers towards dengue in this case were the history of foreign travel, severe thrombocytopenia, marked coagulopathy, and hypotension with mucosal surface bleeding. The presence of dengue virus IgM favored the diagnosis of hepatic failure secondary to DSS. DF should be part of initial differential diagnosis in dealing with patients with acute liver failure and history of foreign travel.

The treatment is supportive in form of fluid replacement, correction of coagulopathy, ventilatory and circulatory support. Therefore, it is plausible to suggest that dengue can be one of the differential diagnoses in acute liver failure, especially in those with recent travel to endemic areas. It is worth mentioning that the incidence of dengue is rapidly increasing.

\section{References}

1. Kyle JL, Harris E. Global spread and persistence of dengue. Annu Rev Microbiol. 2008;62:71-92.

2. WHO, Dengue and severe dengue. Fact sheet no 117. http://www.who.int/mediacentre/factsheets/fs117/en/ Nov 2012

3. Guzman MG, Halstead SB, Artsob H, Buchy P, Farrar J, Gubler DJ, Hunsperger E, et al. Dengue: a continuing global threat. Nat Rev Microbiol. 2010;8(12 Suppl):S7-16.

4. Ranjit S, Kissoon N. Dengue hemorrhagic fever and shock syndromes. Pediatr Crit Care Med. 2011;12(1):90100.

5. Neglected Tropical Diseases. Diseases Covered by NTD department. World Health Organization. Retrieved 201012-27.

6. Gubler DJ. The global emergence/resurgence of arboviral diseases as public health problems. Arch Med Res. 2002;33(4):330-342.

7. Wilder-Smith A, Schwartz E. Dengue in travelers. N Engl J Med. 2005;353(9):924-932.

8. Seneviratne SL, Malavige GN, de Silva HJ. Pathogenesis of liver involvement during dengue viral infections. Trans R Soc Trop Med Hyg. 2006;100(7):608-614.

9. Communicable Disease Surveillance and Response. Clinical Diagnosis. Dengue Haemorrhagic Fever Diagnosis, Treatment, Prevention and Control. 2nd ed. Geneva. World health organisation. 1997. p. 12-23.

10. Nguyen TL, Nguyen TH, Tieu NT. The impact of dengue haemorrhagic fever on liver function. Res Virol. 1997;148(4):273-277.

11. Shah I. Dengue and liver disease. Scand J Infect Dis. 2008;40(11-12):993-994.

12. Parkash O, Almas A, Jafri SM, Hamid S, Akhtar J, Alishah H. Severity of acute hepatitis and its outcome in patients with dengue fever in a tertiary care hospital Karachi, Pakistan (South Asia). BMC Gastroenterol. 2010;10:43.

13. Dengue - an infectious disease of staggering proportions. Lancet. 2013;381(9884):2136. 